\title{
BMJ Open Budget impact analysis of heparin- bonded polytetrafluoroethylene grafts (Propaten) against standard polytetrafluoroethylene grafts for below-the-knee bypass in patients with critical limb ischaemia in France
}

Simon Vergnaud, ${ }^{1,2}$ Valéry-Pierre Riche, ${ }^{1}$ Philippe Tessier, ${ }^{1,3}$ Nicolas Mauduit, ${ }^{2}$ Adrien Kaladji, ${ }^{4}$ Yann Gouëffic ${ }^{4,5,6}$

To cite: Vergnaud S, Riche V-P, Tessier $\mathrm{P}$, et al. Budget impact analysis of heparin-bonded polytetrafluoroethylene grafts (Propaten) against standard polytetrafluoroethylene grafts for below-the-knee bypass in patients with critical limb ischaemia in France. BMJ Open 2018;8:e017320. doi:10.1136/ bmjopen-2017-017320

- Prepublication history for this paper is available online. To view these files, please visit the journal online (http://dx.doi. org/10.1136/bmjopen-2017017320).

Received 18 April 2017 Revised 5 December 2017 Accepted 19 December 2017

Check for updates

For numbered affiliations see end of article.

Correspondence to

Yann Gouëffic;

yann.goueffic@chu-nantes.fr

\section{ABSTRACT}

Objectives To evaluate the budget impact of progressive replacement of standard polytetrafluoroethylene (PTFE) grafts by heparin-bound PTFE (Propaten) for below-the-knee (BTK) bypass in patients with critical limb ischaemia (CLI). Design From a review of the scientific literature, we calculated a theoretical BTK primary patency for Propaten grafts. Using the French hospital expenditure database (PMSI), we retrospectively estimated a rehospitalisation rate for standard PTFE grafts. From these data, a model was created to assess the budget impact of a progressive replacement from standard PTFE grafts to Propaten grafts over a 5-year horizon. We performed an univariate sensitivity analysis to assess the robustness of our results. Setting French National Health Insurance (FNHI) perspective. Participant Patients with CLI.

Main outcome measures Budget impact analysis. Results Data extraction from the PMSI revealed that 656 patients were treated with PTFE grafts in 2011 in French public hospitals for a BTK bypass. Assuming a 2-year survival rate of $76.8 \%$, observed reinterventions rate for standard PTFE grafts at 24 months from the PMSI was $35.1 \%$. The mean rehospitalisation cost was $€ 10689$. The budget impact analysis based on these data found a net cumulative 5-year payer budget reduction of $€ 112420$ in favour of Propaten, under the assumption of a $75.6 \%$ primary patency for Propaten grafts for a projected population of 3215 patients of which 801 received a Propaten graft. Conclusions Our budget impact analysis showed a positive impact on the national health insurance budget of the replacement of standard PTFE grafts by Propaten grafts for BTK bypass in patients with CLI in France. This supports the enactment of a reimbursement policy by the FNHI.

\section{INTRODUCTION}

Patients with critical limb ischaemia (CLI) are at risk of limb amputation. Consequently, a revascularisation should be performed as soon as possible in order to save the limb.

\section{Strengths and limitations of this study}

- The budget impact analysis provides further evidence to adopt and to reimburse the device for decision-makers.

- PMSI database allows for studies with exhaustive data on the French population, thus producing results with a high statistical power and negligible sampling fluctuations.

- However,only patients with critical limb ischaemiaand initially treated by standard polytetrafluoroethylene in public hospital could be identified in the PMSI database, underestimating the results of the study.

- Clinical factors potentially influencing patterns of practice, office-based consumption of cares, and non-reimbursable items and medicines could not be analysed.

To realise the revascularisation, two options should be considered: endovascular or open repair. So far, despite the lack of consensus, open repair could be recommended in a first line of treatment to revascularise patients with $\mathrm{CLI}^{2}$ or performed in a second line of treatment in case of failure of endovascular repair. ${ }^{3}$ In the event of open surgery, a vein should be used as conduit to perform the bypass, especially in the case of infrapopliteal lesions. A suitable vein is one of the main factors that determine the clinical success of open revascularisation for below-the-knee (BTK) popliteal and distal bypass. ${ }^{1}$ Unfortunately, a suitable venous conduit is not available in more than $20 \%$ of the cases. ${ }^{2}$ In these patients, prosthesis such as standard polytetrafluoroethylene (PTFE) graft demonstrated worse clinical and morphological results and more severe consequences in 
case of occlusion. ${ }^{45}$ Consequently, there is still a room for improvement in patients with CLI in the absence of a suitable conduit and in whom endovascular repair failed. In these patients, prostheses with heparin-bound to the luminal surface could improve standard prosthesis results. In 2011, Lindholt et at reported the results of a multicentre randomised trial comparing heparin-bound PTFE (Propaten, Flagstaff, AZ, USA) grafts with those of standard PTFE grafts. In total, 546 patients had 1-year follow-up (standard PTFE: 272; Propaten: 274). Propaten graft significantly reduced the overall risk of primary graft failure by $37 \%$ at 1 year from the intervention. Specifically, risk reduction reached $50 \%$ in femoropopliteal bypass for patients with CLI. Moreover, after 5 years, patients receiving Propaten grafts for CLI were more likely to have a patent graft than those with standard PTFE grafts. ${ }^{7}$

However, to date, the financial impact of Propaten use on healthcare spending was not assessed. Using data from the literature and from the French hospital expenditure database (PMSI), we assess the financial impact of a progressive replacement of standard PTFE by Propaten on a 5-year timeline from the payer perspective, for BTK bypass in patients with CLI.

\section{METHODS}

\section{Analytic overview}

Our aim was to compare the usual course of action taken by French surgeons for BTK bypass surgery, using standard PTFE grafts, to a similar course of action using Propaten grafts, in order to assess the latter's economical impact. We combined clinical data based on a review from the literature and retrospective data about hospital stays from the PMSI to feed a cost model from a third party payer perspective and to perform a budget impact analysis. No change in our clinical practice and no randomisation occurred. As our model was based on an observational retrospective analysis of data, according to the French legislation (articles L.1121-1 paragraph 1 and R1121-2, Code de la Santé Publique), approval of an ethics committee was not required for use of the data in an epidemiological study.

\section{Evidence acquisition}

Our search strategy was based on Preferred Reporting Items for Systematic Reviews and Meta-analyses (PRISMA) guidelines, with the help of PRISMA statement and explanation and elaboration documents. ${ }^{8}$ We used MEDLINE register to conduct our bibliography. The following terms were added to the search builder using MeSH: below the knee, bypass, surgery, Propaten ${ }^{\circledR}$, grafts, 2years, primary patency and critical limb ischemia. One study was excluded because it focused exclusively on diabetics. ${ }^{9}$ Another was found to have an outlying rate of renal insufficiency. ${ }^{10}$ Indeed, we considered that outlying rate of diabetes and renal insufficiency could alter too much the outcomes in regards to perioperative outcomes and pattern of atherosclerotic disease. ${ }^{11} 12$ We assigned each study a weight, assuming a fixed-effect model (table 1). Our estimate of the 2-year BTK primary patency for Propaten grafts was $75.6 \%$, ranging from $70.8 \%$ to $85 \%$.

\section{Source population}

From a retrospective analysis on hospital stays during 2011 using the PMSI, we identified patients who were admitted for a BTK bypass surgery, where a standard PTFE graft was used. In France, only patients with CLI have this surgery, where using a standard PTFE graft is the usual choice (French medical information agencyATIH-online data). ${ }^{13}$ Therefore, no analysis was conducted on other types of grafts. Propaten grafts were not available in France in 2011. Patients under 18 years old were excluded since bypasses in this population are not indicated to treat an atheromatous disease but to revascularise a lower limb for an inflammatory arterial disease or an arterial traumatism. Patients having been

Table 1 Detailed review of the literature of the Propaten patency rate at 2 years

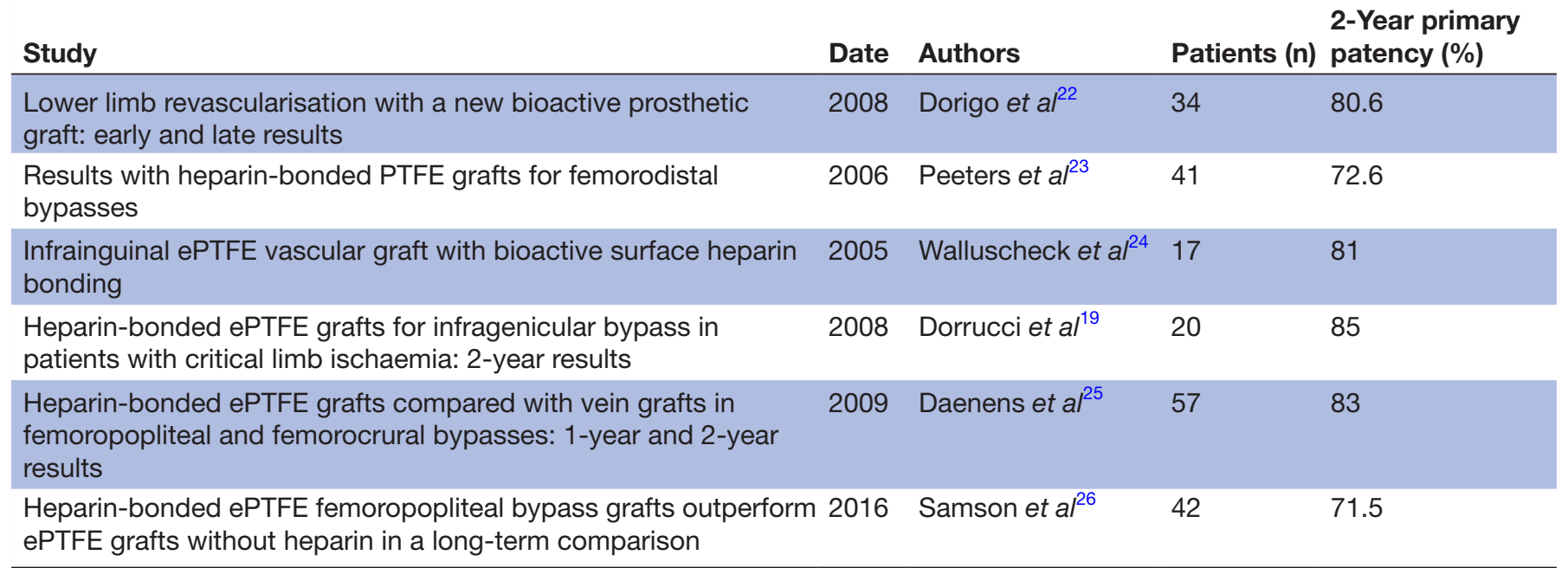

ePTFE, expanded polytetrafluoroethylene; PTFE, polytetrafluoroethylene. 


\begin{tabular}{|c|c|c|}
\hline Clinical data & Values & Sources \\
\hline First rehospitalisation rate due to graft of interest & $\begin{array}{l}35.1 \% \\
(177 / 504)\end{array}$ & $\begin{array}{l}\text { French rehospitalisation data, adjusted for } \\
\text { mortality }^{16} \text { and contralateral reintervention }{ }^{15}\end{array}$ \\
\hline Added primary patency for Propaten grafts: & $75.6 \%$ & Own calculations (evidence acquisition) \\
\hline \multicolumn{3}{|l|}{ Cost estimates } \\
\hline Propaten initial additional cost & $€ 627$ & WL Gore \\
\hline PTFE reimbursement tariff & $€ 639$ & FNHI online data ${ }^{13}$ \\
\hline \multicolumn{3}{|l|}{ Market data } \\
\hline Initial market penetration & $15 \%$ & De Cock ${ }^{17}$ \\
\hline
\end{tabular}

FNHI, French National Health Insurance; PMSI, French hospital expenditure database; PTFE, polytetrafluoroethylene.

operated on for a BTK bypass surgery in the 2 years prior were excluded in order to exclude reinterventions from index cases. The data included the reference of the diagnosis-related groups (DRGs), the type of bypass grafts used, the duration of stays, the time spent in intensive care unit and the patient's comorbidities. Patients were followed for 24 months. ${ }^{14}$

\section{The population model}

First of all, the follow-up of the source population was determined. Rehospitalisation in relation to the standard PTFE was determined by a retrospective analysis on hospital stays for our source population during the 24 months following the initial surgery. The follow-up of the source population was adjusted for 2-year mortality and contralateral reintervention. ${ }^{15} 16$ Loss of patency was defined by a hospital stay for a lower limb reintervention hereafter called the first rehospitalisation. These lower limb interventions included angioplasties, major amputations, thrombectomies, ablations of vascular grafts, stent placements and in situ fibrinolysis (table 2).

\section{Retrospective cost estimation for standard PTFE grafts}

As we aimed to estimate the budget impact of an official reimbursement policy, we conducted our budget impact analysis from the payer perspective (French National Health Insurance, FNHI) and estimated costs only from this perspective. Only direct medical costs, covering in-patient treatment, were considered. Costs were estimated by the 2015 official tariffs applied to the relevant DRGs, for both initial and further hospitalisations. The tariffs provide the amount paid by the FNHI to a hospital with respect to each stay, procedure duration and potential additional costs, that is, hospital costs that are reimbursed in addition to the DRG tariff (eg, intensive care). Variability was estimated for both initial and subsequent interventions using a bootstrap technique, with a resampling of 100 random samples of 100 patients.

\section{Cost estimation for Propaten grafts}

We estimated the costs for the initial Propaten procedure using the mean initial intervention cost (MIIC) for standard PTFE grafts added to the cost difference (€627) between Propaten graft's market price and the reimbursed tariff for standard PTFE grafts. The mean rehospitalisations cost (MRC) for standard PTFE was used to estimate the mean cost for Propaten rehospitalisations. Every bypass graft used during rehospitalisation stays was assumed to be a standard PTFE graft.

\section{Budget impact model}

Our budget impact analysis premised the enactment by the French Health Authorities of a FNHI reimbursement policy, that is, additional costs from the initial procedure would be charged solely to the FNHI. Our base case for the budget impact model used the estimates from our literature review to estimate a rehospitalisation rate for the Propaten implantation for the 2011 PMSI-extracted population. No analysis was conducted on 2-year secondary patency mainly because of the lack of PMSI data on limb side. Total hospital reimbursement costs for both procedures were calculated by adding the initial intervention costs with subsequent rehospitalisations costs. Each year for 5 years, a new cohort of patients entered the model for a duration of 2 years, starting with the 2011 population. The number of patients decreases by a flat $1.0 \%$ annually, that is, the mean decrease rate between 2011 and 2014 for the DRG representing 95\% of our population as informed in ATIH online data. ${ }^{13}$ We hypothesised that the enactment of a reimbursement policy by French Health authorities would result in an initial market penetration rate of $15 \%$ for Propaten grafts, with a subsequent annual increase of $5 \%$ points, meaning that after 5 years, $35 \%$ of the grafts in this indication would be Propaten grafts. ${ }^{17}$ Numerical values corresponding to the hypotheses we made are presented in table 2 . We based our sensitivity analysis on variation one by one of relevant variables 
in order to assess the weight of each hypothesis on the overall behaviour of the model. ${ }^{18}$

\section{Sensitivity analysis}

Univariate analysis

We tested the sensitivity of our results to the main hypotheses used in our model by estimating the budget impact for a range of values. The tested parameters were the 2-year BTK primary patencies for Propaten and standard PTFE grafts, the mean cost of rehospitalisation and the additional cost of the initial intervention for Propaten grafts. For Propaten grafts' patency, we used the 2-year primary patency for standard PTFE grafts found in our population as low end of the range, and the highest reported primary patency ${ }^{19}$ as high end. For PTFE grafts' patency, we used the 2-year primary patency for standard PTFE grafts found in our population as the high end of the range, and the value found in the literature ${ }^{4}$ as the low end. For the mean cost of rehospitalisations, we used the low and high values of our $95 \% \mathrm{CI}$ as low and high ends of the range. Finally, we used arbitrary values to test for the sensitivity of our results to the price of the Propaten graft.

\section{Scenario analysis}

We estimated the 5-year budget impact in three additional scenarios, describing one alternative plausible situation and the two extremes. These extreme scenarios are described in table 3, and either favoured (best case) or disfavoured (worst case) Propaten grafts, based on 2-year BTK primary patency for Propaten grafts, mean cost of rehospitalisations and 2-year BTK primary patency for standard PTFE grafts. The alternative plausible scenario assumed that a maximum patency would decrease the mean cost of rehospitalisations.

\section{RESULTS}

\section{Retrospective database analysis for standard PTFE grafts}

The retrospective data from the national expenditure database revealed 656 patients with CLI treated with standard PTFE grafts for a BTK bypass surgery in 2011. Two years later, 152 patients had died and 504 patients were still alive. Among these 504 patients, 189 patients had been
Table 3 Worst and best case of the scenario analysis

\begin{tabular}{llll}
\hline & & & Worst \\
& Base case (\%) & Best case (\%) & $\begin{array}{l}\text { case (\%) } \\
\text { case }\end{array}$ \\
\hline PTFE & 64.9 & 47.0 & 64.9 \\
Propaten & 75.6 & 85.0 & 64.9 \\
\hline
\end{tabular}

PTFE, polytetrafluoroethylene.

re-hospitalised at 2years. From the literature, ${ }^{15} 2021$ we estimated that 12 of these patients had had interventions on the contralateral limb only. Consequently, 177 patients were hospitalised at 2 years for a BTK surgical intervention on the limb of interest, resulting in a rehospitalisation rate of $35.1 \%$, or a 2-year primary patency of $64.9 \%$ for standard PTFE grafts. In the assumed group (treatment with Propaten grafts) the estimated patency rate was $75.6 \%$ at 2 years (table 1) and we predict 123 rehospitalisations for the Propaten grafts group.

\section{Costs of treatment using standard PTFE and Propaten}

The MIIC from the payer perspective was $€ 12290$ (95\% CI $€ 11118$ to $€ 13$ 386) per patient (total initial intervention costs: $€ 8062$ 382). Most patients (99\%) belonged to the DRG for major revascularisation surgeries (DRG 05C10). The MRC from the payer perspective was $€ 10$ 689 (95\% CI $€ 9464$ to $€ 12072)$. Two-year total hospitalisation cost from the national insurance perspective for the 656 patients with standard PTFE grafts was $€ 9$ 008 321. Assuming treatment with only Propaten grafts for the 656 patients from 2011, 2-year total hospital reimbursement costs would have been €9 130998 .

\section{Budget impact analysis}

Under the base case assumptions (table 2), we calculated a difference in MIIC of $€ 502173$ in favour of standard PTFE grafts over a 5 -year period (table 4 ).

We projected a cumulative population of 3215 patients over 5 years, of which 801 would have received a Propaten graft. At 5 years, we would have avoided 57 rehospitalisations, resulting in cost reduction of $€ 614593$ in favour of Propaten grafts. The amount of savings due to fewer rehospitalisations offset the difference in MIIC as soon

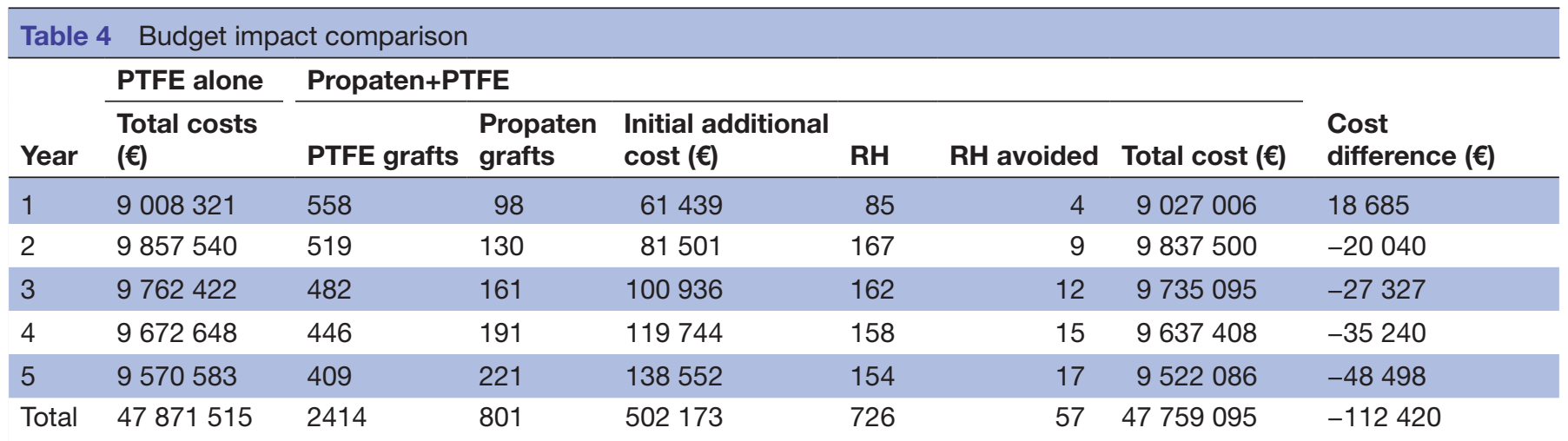

Plus sign indicates an increase in costs, and minus sign indicates savings.

PTFE, polytetrafluoroethylene; $\mathrm{RH}$, rehospitalisation. 


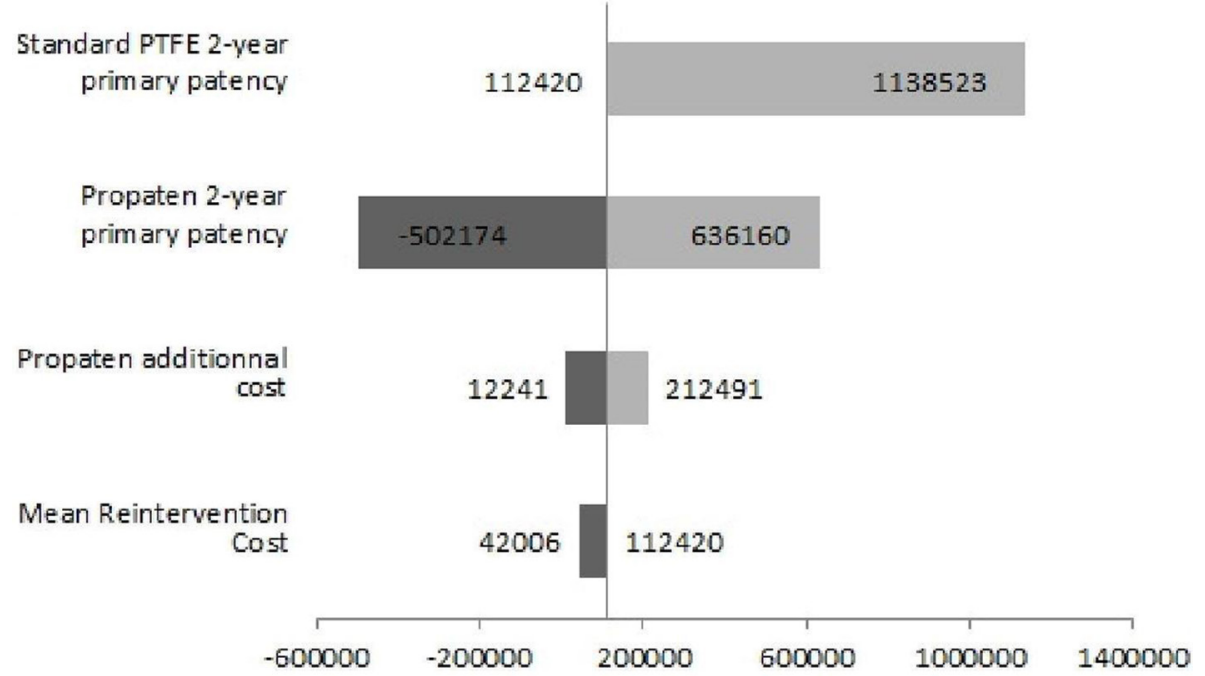

Figure 1 Tornado diagram representing the variation of the 5-year budget balance depending on five hypotheses. A negative balance indicates a cost-save. Costs are in euros $(€)$. Positive costs indicate savings, negative costs indicate a cost increase. PTFE, polytetrafluoroethylene.

as the second year. Assuming a $15 \%$ market penetration during the first year and then $5 \%$ fixed market penetration ( $35 \%$ over the 5 years), the total difference between the observed standard PTFE and assumed Propaten+PTFE courses was estimated at $€ 112420$, in favour of Propaten grafts, from the FNHI perspective.

\section{Sensitivity analysis}

Univariate analysis

Primary patency for Propaten had a strong impact on budget results. Using the lower rate of primary patency at 2 years (worst case), the additional cost was $€ 486140$. On the contrary, using the higher patency rate (best case), the saving was $€ 636160$. For PTFE grafts, a primary patency closer to the values found in the literature $(47 \%)$ increased the savings allowed by Propaten grafts. ${ }^{4}$ The market price for Propaten grafts (initial intervention additional cost) had comparatively little impact on the 5-year budget balance and so did MRC when including further rehospitalisations. A cheaper graft or a higher MRC led to higher 5-year savings(figure 1).

\section{Scenario analysis}

Our worst and best cases showed the variability of the budget impact of Propaten grafts with a difference of more than $€ 2.4$ million (table 5 ).

\section{DISCUSSION}

Our model-based analysis showed the 5-year budget impact for the diffusion of Propaten in replacement of standard PTFE to be cost-saving. This is a strong economic incentive in favour of both a widespread use and the enactment of a reimbursement policy for Propaten grafts.

Our modelling approach was founded on a set of assumptions that deserve mention.

The centralised structure of the French Health Information system allows for low-cost studies with exhaustive data on the French population, thus producing results with a high statistical power and negligible sampling fluctuations.

Few articles on Propaten grafts presented 2-year primary patency for BTK bypasses in the general population, and the level of their clinical evidence was limited. We excluded two articles because of the epidemiological profile (diabetes and renal failure) of their populations, which were associated with higher morbimortality and lower patency rates overall. Usually patients with BTK bypasses represent a homogeneous group of patients with critical limb ischaemia in comparison to above the knee bypass, which could be realised for claudicants or patients with CLI. Furthermore, no article presented specific results on BTK bypasses in patients who were critically ischaemic, and two articles had better outcomes for BTK than above-the-knee revascularisation. This usually is not the case in lower limb bypasses, and could be partially explained by important sampling fluctuations due to their small sample sizes. As there was no other available data, we chose to use reasonably unfavourable hypotheses in our analyses to compensate for these shortcomings and thus strengthen the overall conclusion. Nevertheless, as we excluded those two studies, our results should only

\begin{tabular}{|c|c|c|c|}
\hline & Base case & Best case & Worst case \\
\hline PTFE & $64.9 \%$ & $47.0 \%$ & $64.9 \%$ \\
\hline Propaten & $75.6 \%$ & $85.0 \%$ & $64.9 \%$ \\
\hline MRC & $€ 10689.00$ & $€ 12072.00$ & $€ 9464.00$ \\
\hline $\begin{array}{l}\text { 5-Year } \\
\text { budget } \\
\text { impact }\end{array}$ & $-€ 112419.75$ & $€ 1942406.40$ & $-€ 502173.60$ \\
\hline
\end{tabular}

MRC, mean rehospitalisation cost; PTFE, polytetrafluoroethylene. 
be considered valid for unselected CLI populations. In such populations, diabetes, although frequent, rarely has a $100 \%$ prevalence rate, and renal insufficiency rates are about half the rate from the Lösel-Sadée study $(31.0 \%){ }^{9}$

As our sensitivity analysis showed, our conclusions are tied to both the effectiveness of Propaten grafts and the comparative lack of effectiveness of standard PTFE grafts. The observed 2-year primary patency for standard PTFE grafts is about $35 \%$ higher than usually described. ${ }^{4}$ Most clinical studies follow their patients more thoroughly than it is the case in daily care. This is the cause of a follow-up bias in our study, due to the use of reintervention as a measure of loss of patency, which overestimate the patency for standard PTFE grafts. Indeed, in the case of an occluded graft, reintervention and/or amputation are not systematically performed because the patient is asymptomatic or because a palliative treatment is decided. These types of health consumptions are not logged in the PMSI database and as we used intervention-specific codes, we estimated the 2-year primary patency for standard PTFE grafts using only patients with lower limb vascular surgical interventions. The patients lost because of our method of follow-up would only ramp up the costs of the standard PTFE course of action.

We used hospital reimbursement costs only, as they are likely to be cost-drivers in a surgical course of action. Unavailable costs included those for non-hospital medical consultations and care, which are likely to decrease with a more effective Propaten graft. Likewise, the exclusion of readmissions past the first one may only have lessened the difference in costs between the two types of grafts. It was anyhow not an option to use these readmissions, given the uncertainty on limb side and the lack of available data.

Our scenario analysis showed the extent of the potential budget impact that would follow the globalisation of Propaten use for BTK bypasses in France. Unfortunately, the uncertainty around the 2-year primary patency translated to an extensive range for its budget impact. The worst-case scenario assumed that Propaten grafts were no more effective than standard PTFE grafts in our population, which is pessimistic, but for which the likelihood does not seem quantifiable.

Finally, even though we based our model on French data and tariffication, it can be used for any DRG-based system to estimate the budget impact of Propaten reimbursement.

\section{CONCLUSION}

At current times of resource allocation rationalisation, every innovation in healthcare must pass tests of both clinical and economic value. Propaten grafts have shown their clinical effectiveness, but had yet to be proven economically attractive.

In this paper, we used existing clinical proof to show that Propaten grafts in patients with CLI needing a BTK bypass would be financially beneficial for the FNHI in most cases. The decision to specifically reimburse Propaten at its market price dictates the extent of its use throughout France, as few hospitals can afford it in a DRG-based system, which does not allow them to benefit directly from the increased primary patency. Based on our hypotheses and analysis, we conclude that a reimbursement policy would benefit both the FNHI and the patients. Our model allows performing the same analysis in other countries using local cost and clinical effectiveness data providing they have a similar reimbursement system.

Future research ought to focus on directly comparing standard PTFE and Propaten grafts in order to confirm its probable cost-effectiveness dominance.

\section{Author affiliations}

${ }^{1}$ Innovation Cell, Partnership and Innovation Department, Directorate of Medical Affairs and Research, CHU de Nantes, Nantes, France

${ }^{2}$ Department of Medical Information, CHU de Nantes, Nantes, France

${ }^{3}$ SPHERE (EA4275) - Biostatistics, Clinical Research and Pharmaco-Epidemiology, Nantes University, Nantes, France

${ }^{4}$ Service de Chirurgie Vasculaire, CHU Nantes, l'institut du Thorax, Nantes, France ${ }^{5}$ Laboratoire de Physiopathologie de la Résorption Osseuse, Inserm-UN UMR-957, Nantes, France

${ }^{6}$ Faculté de médecine, Université de Nantes, Nantes, France

Acknowledgements The data extracted from the PMSI was supplied by the French Medical Information Agency (ATIH) and was used under agreement with the French data protection authority (CNIL) with the authorisation numbers DE-2011066 and 2015-064. Only authorised and discretion-bound personnel handled this data. The model was created using Microsoft Excel.

Contributors SV, V-PR and PT: conception and design, analysis and interpretation, data collection, writing the manuscript, critical revision of the manuscript and statistical analysis. NM: analysis and interpretation, data collection, writing the manuscript and critical revision of the manuscript. AK: analysis and interpretation, writing the manuscript and critical revision of the manuscript. YG: conception and design, analysis and interpretation, writing the manuscript, critical revision of the manuscript and obtaining funding.

Funding This research was carried out with financial support from WL Gore.

Competing interests YG received consulting fees and travel grant from Abbott, Bard, Biotronik, Boston Sc, Cook, Medtronic, Perouse, Spectranetics, Terumo and WL Gore.

Patient consent Not required.

Provenance and peer review Not commissioned; externally peer reviewed. Data sharing statement No additional data available.

Open Access This is an Open Access article distributed in accordance with the Creative Commons Attribution Non Commercial (CC BY-NC 4.0) license, which permits others to distribute, remix, adapt, build upon this work non-commercially, and license their derivative works on different terms, provided the original work is properly cited and the use is non-commercial. See: http://creativecommons.org/ licenses/by-nc/4.0/

(C) Article author(s) (or their employer(s) unless otherwise stated in the text of the article) 2018. All rights reserved. No commercial use is permitted unless otherwise expressly granted.

\section{REFERENCES}

1. Norgren L, Hiatt WR, Dormandy JA, et al. Inter-society consensus for the management of peripheral arterial disease (TASC II). Eur J Vasc Endovasc Surg 2007;33 Suppl 1:S1-75.

2. Anderson JL, Halperin JL, Albert NM, et al. Management of patients with peripheral artery disease (compilation of 2005 and 2011 ACCF/ AHA guideline recommendations): a report of the American College 
of Cardiology Foundation/American Heart Association Task Force on Practice Guidelines. Circulation 2013;127:1425-43.

3. Setacci C, de Donato G, Teraa M, et al. Chapter IV: Treatment of critical limb ischaemia. Eur J Vasc Endovasc Surg 2011;42(Supplement 2):S43-59.

4. Albers M, Battistella VM, Romiti M, et al. Meta-analysis of polytetrafluoroethylene bypass grafts to infrapopliteal arteries. $J$ Vasc Surg 2003;37:1263-9.

5. Jackson MR, Belott TP, Dickason T, et al. The consequences of a failed femoropopliteal bypass grafting: comparison of saphenous vein and PTFE grafts. J Vasc Surg 2000;32:498-505

6. Lindholt JS, Gottschalksen B, Johannesen N, et al. The Scandinavian

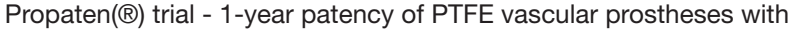
heparin-bonded luminal surfaces compared to ordinary pure PTFE vascular prostheses - a randomised clinical controlled multi-centre trial. Eur J Vasc Endovasc Surg 2011;41:668-73.

7. Lindholt JS, Houlind K, Gottschalksen B, et al. Five-year outcomes following a randomized trial of femorofemoral and femoropopliteal bypass grafting with heparin-bonded or standard polytetrafluoroethylene grafts. Br J Surg 2016;103:1300-5.

8. Moher D, Liberati A, Tetzlaff J, et al. Preferred reporting items for systematic reviews and meta-analyses: the PRISMA statement. PLoS Med 2009;6:e1000097.

9. Dorigo W, Pulli R, Castelli P, et al. A multicenter comparison between autologous saphenous vein and heparin-bonded expanded polytetrafluoroethylene (ePTFE) graft in the treatment of critical limb ischemia in diabetics. $J$ Vasc Surg 2011;54:1332-8.

10. Lösel-Sadée H, Alefelder C. Heparin-bonded expanded polytetrafluoroethylene graft for infragenicular bypass: five-year results. J Cardiovasc Surg 2009;50:339-43.

11. Diehm N, Shang A, Silvestro A, et al. Association of cardiovascular risk factors with pattern of lower limb atherosclerosis in 2659 patients undergoing angioplasty. Eur J Vasc Endovasc Surg 2006;31:59-63.

12. Wasmuth $\mathrm{S}$, Baumgartner I, Do DD, et al. Renal insufficiency is independently associated with a distal distribution pattern of symptomatic lower-limb atherosclerosis. Eur J Vasc Endovasc Surg 2010;39:591-6.

13. Statistics from the French Medical Information Agency (ATIH). ScanSanté. http://www.scansante.fr/. (cited 8 Jan 2016).

14. Conte MS. Understanding objective performance goals for critical limb ischemia trials. Semin Vasc Surg 2010;23:129-37.
15. de Vries SO, Donaldson MC, Hunink MG. Contralateral symptoms after unilateral intervention for peripheral occlusive disease. J Vasc Surg 1998;27:414-21.

16. Martini R, Andreozzi GM, Deri A, et al. Amputation rate and mortality in elderly patients with critical limb ischemia not suitable for revascularization. Aging Clin Exp Res 2012;24(3 Suppl):24-7.

17. De Cock E, Sapoval M, Julia P, et al. A budget impact model for paclitaxel-eluting stent in femoropopliteal disease in France. Cardiovasc Intervent Radiol 2013;36:362-70.

18. Sullivan SD, Mauskopf JA, Augustovski F, et al. Budget impact analysis-principles of good practice: report of the ISPOR 2012 budget impact analysis good practice II task force. Value Health 2014;17:5-14.

19. Dorrucci V, Griselli F, Petralia G, et al. Heparin-bonded expanded polytetrafluoroethylene grafts for infragenicular bypass in patients with critical limb ischemia: 2-year results. 2008:49-2.

20. Shah AP, Klein AJ, Sterrett A, et al. Clinical outcomes using aggressive approach to anatomic screening and endovascular revascularization in a veterans affairs population with critical limb ischemia. Catheter Cardiovasc Interv 2009;74:11-19.

21. Liistro F, Porto I, Angioli P, et al. Drug-eluting balloon in peripheral intervention for below the knee angioplasty evaluation (DEBATEBTK): a randomized trial in diabetic patients with critical limb ischemia. Circulation 2013:128:615-21.

22. Dorigo W, Di Carlo F, Troisi N, et al. Lower limb revascularization with a new bioactive prosthetic graft: early and late results. Ann Vasc Surg 2008;22:79-87.

23. Peeters P, Verbist J, Deloose K, et al. Results with heparin bonded polytetrafluoroethylene grafts for femorodistal bypasses. $J$ Cardiovasc Surg 2006;47:407-13.

24. Walluscheck KP, Bierkandt S, Brandt M, et al. Infrainguinal ePTFE vascular graft with bioactive surface heparin bonding. First clinical results. J Cardiovasc Surg 2005;46:425-30.

25. Daenens K, Schepers S, Fourneau I, et al. Heparin-bonded ePTFE grafts compared with vein grafts in femoropopliteal and femorocrural bypasses: 1- and 2-year results. J Vasc Surg 2009;49:1210-6.

26. Samson RH, Morales R, Showalter DP, et al. Heparin-bonded expanded polytetrafluoroethylene femoropopliteal bypass grafts outperform expanded polytetrafluoroethylene grafts without heparin in a long-term comparison. J Vasc Surg 2016;64:638-47. 